\title{
"Cinematic rendering" of an Egyptian fish mummy with a fractured spinal column
}

\section{Franz A. Fellner, ${ }^{1, *}$}

${ }^{1}$ Central Radiology Institute, Kepler University Hospital, Medical Faculty of the Johannes Kepler University, Linz, Austria

${ }^{2}$ Medical Faculty of the Friedrich-Alexander-University of Erlangen-Nürnberg, Germany

The ancient Egyptians mummified their dead in preparation for the afterlife. And this was performed not only on human beings; animals too were embalmed to accompany humans into the afterlife as, so to speak, mummified food or pets. The form of mummification and the mode of wrapping the corpse in linen bandages can provide indications as to the decedent's age. Thus, for centuries, researchers eager to unveil the secret of what lay beneath the wrappings removed the bandages and, in the process, destroyed the mummy or severely damaged it at the very least. Today, $\mathrm{x}$-ray and computed tomography (CT) technology make it possible to analyze mummies without ruining them. We were recently provided access to an approximately 2,000-year-old mummified fish from the collection of the Austrian National Bank for the purpose of non-destructive analysis by means of a routine clinical CT scanner.

We then used the CT data set to generate three-dimensional depictions by means of the prototype of a new post-processing 3D rendering method ("Cinematic Rendering") we recently presented [1]. The Cinematic Rendering software makes it possible to produce such images in photorealistic quality.

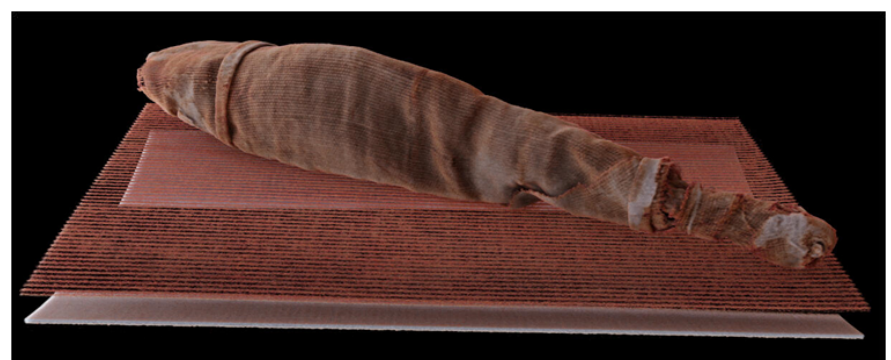

Figure 1. Three-dimensional surface reconstruction of the CT scan of the bandaged fish mummy using Cinematic Rendering.

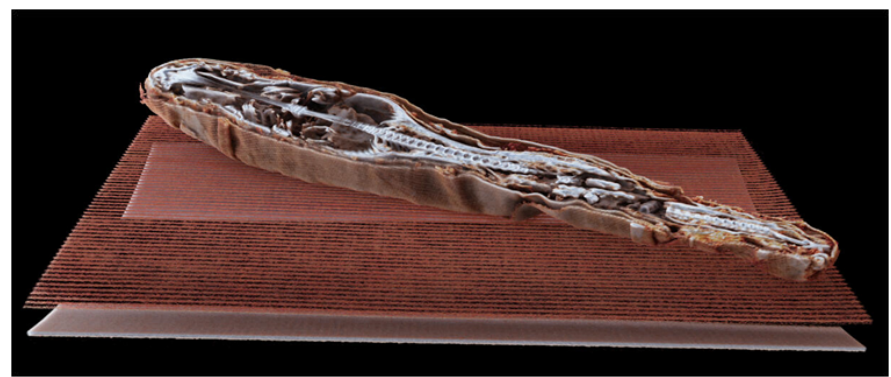

Figure 2. Cinematic Rendering of the CT scan. Here, the mummy has been virtually opened. The skeletal system is quite well preserved on the whole; nevertheless, the skeleton is damaged in the transitional zone from the midsection to the tail, which is most obviously apparent in its fractured spinal column.

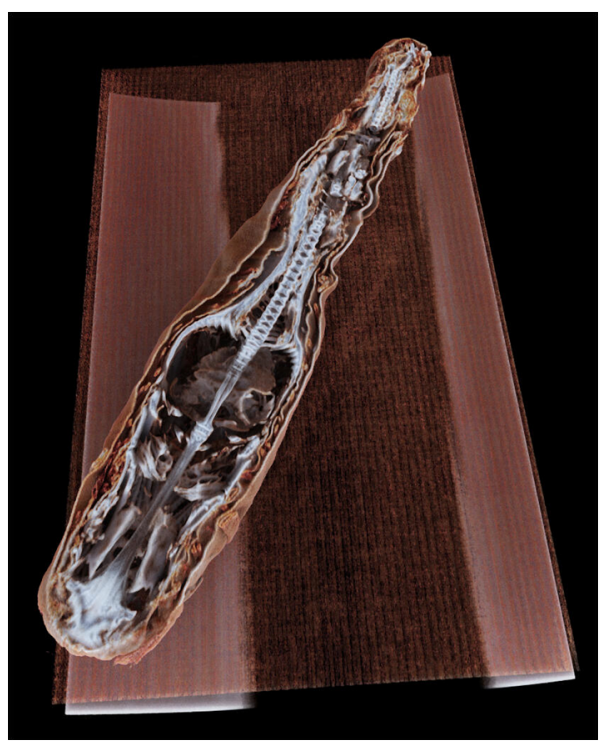

Figure 3. Cinematic Rendering of the CT scan. A view down onto the virtually opened mummy.

Correspondence to: Franz A. Fellner, Central Radiology Institute, Kepler University Hospital, Medical Faculty of the Johannes Kepler University, Linz, Austria, E-mail: franz.fellner@kepleruniklinikum.at

Received: December 02, 2016; Accepted: December 12, 2016; Published: December 14, 2016 


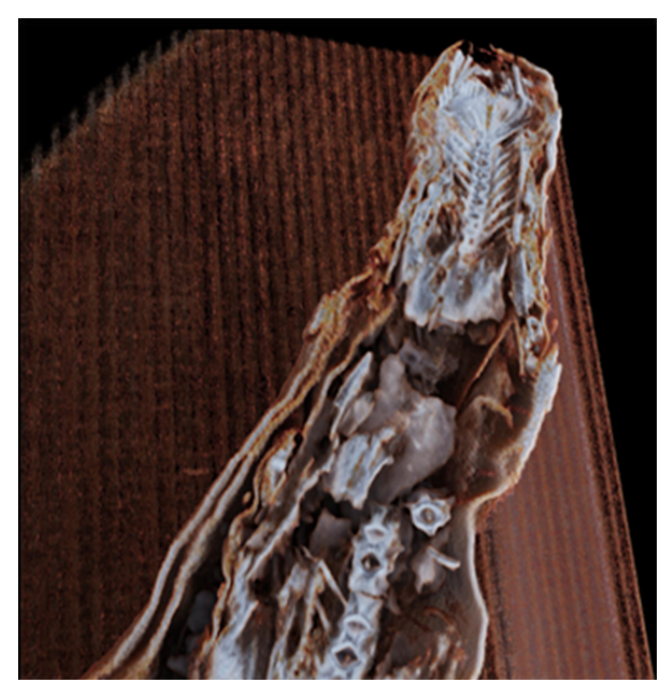

\section{References}

1. Fellner FA (2016) Introducing cinematic rendering: a novel technique for postprocessing medical imaging data. J Biomedical Science and Engineering 9: 170-175.

Figure 4. Cinematic Rendering of the CT scan. Close-up view of the damaged area with fracture of the spinal column; the rearmost section of the skeleton, however, is very well preserved.

Copyright: ( 02016 Fellner FA. This is an open-access article distributed under the terms of the Creative Commons Attribution License, which permits unrestricted use, distribution, and reproduction in any medium, provided the original author and source are credited. 